Колобанова Евгения

Сергеевна - врач-рентгенолог рентгенодиагностического отделения Научно-исследовательского института клинической и экспериментальной радиологии'; ORCID: https://orcid. org/0000-0002-1563-0983

$\triangle 115478$, г. Москва, Каширское шоссе, 23, Российская Федерация. Тел.: +7 (985) 15230 39. E-mail: evgesha.16.kolobanova@gmail.com

Медведева Бэла Михайловна д-р мед. наук, вед. науч. сотр. рентгенодиагностического отделения Научно-исследовательского института клинической и экспериментальной радиологии'; ORCID: https://orcid. org/0000-0003-1779-003X
ФГБУ «Национальный медицинский исследовательский центр онкологии имени Н.Н. Блохина» Минздрава России; 115478, г. Москва, Каширское шоссе, 23, Российская Федерация
Актуальность. Значимой проблемой современных методов визуализации жиросодержащих образований в области брыжейки тонкой кишки остается дифференциальная диагностика между поражениями, в отношении которых необходимо хирургическое вмешательство или медикаментозное лечение.

Цель - выявить дифференциально-диагностические критерии различных жиросодержащих новообразований абдоминальной области на дооперационном этапе.

Материал и методы. В основу работы положены данные ретроспективного исследования 29 пациентов (16 женщин и 13 мужчин в возрасте от 32 до 81 года, средний возраст 56 лет) с жиросодержащими образованиями в области брыжейки тонкой кишки, проходивших предоперационное обследование в ФГБУ «НМИЦ онкологии им. Н.Н. Блохина» Минздрава России в период с 2016 по 2020 г. Всем пациентам выполнялась рентгеновская компьютерная томография (РКТ) органов брюшной полости с внутривенным контрастированием с дальнейшей оценкой размеров, контуров, структуры и вовлечения магистральных сосудов. При анализе отдельных мягкотканных узлов в толще образования мы пользовались разработанным нами перечнем основных компьютерно-томографических проявлений (количество, размеры, форма, контуры и структура узлов, степень их контрастирования и максимальное усиление).

Результаты. Морфологическая верификация жиросодержащих опухолей абдоминальной области была представлена следующим образом: мезентериальный панникулит - 13 (45\%), высокодифференцированная липосаркома 10 (35\%), лимфопролиферативное поражение - 4 (14\%) и по единичному наблюдению липомы (3\%) и болезни Кастлемана (3\%). Из 29 пациентов 12 (41\%) были прооперированы по поводу опухолевых образований различной природы, 17 (59\%) получили соответствующее медикаментозное лечение после проведения пункционной биопсии и верификации процесса. Чувствительность РКТ в выявлении жиросодержащих новообразований абдоминальной области в наших наблюдениях составила 93,1\%. В статье приведены рентгенологические признаки представленных выше разновидностей образований с акцентом на сложности диагностики.

Заключение. Полученные при РКТ данные в большинстве случаев позволяют безошибочно дифференцировать липосаркомы абдоминальной области с другими доброкачественными и злокачественными процессами, расположенными в брыжейке тонкой кишки.

Ключевые слова: липосаркома, мезентериальный панникулит, лимфома, болезнь Кастлемана

Для цитирования: Колобанова ЕС, Медведева БМ. Сложности дифференциальной диагностики неорганных опухолей абдоминальной области. Альманах клинической медицины. 2021;49(1):29-40. doi: 10.18786/2072-0505-2021-49-008.

Поступила 07.12.2020; доработана 26.12.2020; принята к публикации 03.03.2021; опубликована онлайн 15.03.2021 
$\mathrm{H}$

еорганные забрюшинные опухоли редкая патология: среди всех видов сарком мягких тканей на их долю приходится от 10 до 15\% [1]. По данным литературы, из забрюшинных мезенхимальных опухолей наиболее часто встречается липосаркома, составляющая более $50 \%$ от общего числа сарком [2]. В 24\% случаев липосаркомы локализуются на конечностях и туловище, в $45 \%$ - в брюшной и забрюшинной областях [3]. По происхождению липосаркомы относятся к злокачественным мезенхимальным новообразованиям, они состоят из жировой ткани, характеризуются различными уровнями атипии и могут содержать включения саркоматозных клеток нежировой природы [4].

Самый распространенный гистологический тип липосарком - высокодифференцированные липосаркомы. Они могут быть расположены в брыжейке тонкой кишки и часто имитируют другие доброкачественные и злокачественные процессы. Так, в частности, среди злокачественных поражений тканей абдоминальной области можно выделить ряд лимфопролиферативных заболеваний, таких как болезнь Ходжкина и ретикулосаркома, среди поражений неопухолевой природы - мезентериальный панникулит. Последний представляет собой хроническое неспецифическое воспаление жировой ткани брыжейки кишечника, сальника, предбрюшинной и забрюшинной клетчатки [5]. Однако, несмотря на внешнюю схожесть патологических процессов по данным лучевых методов визуализации, имеется ряд семиотических особенностей, способных кардинальным образом повлиять на выбор лечебной тактики и в ряде случаев уберечь пациента от неоправданного хирургического вмешательства.

Насколько нам известно, в мировой литературе данные о дифференциальной диагностике абдоминальных липосарком с другими злокачественными и доброкачественными жиросодержащими новообразованиями представлены немногочисленными наблюдениями [6-13].

Цель исследования - выявить семиотические компьютерно-томографические (КТ)-признаки, позволяющие корректно дифференцировать липосаркому с другими доброкачественными и злокачественными процессами в брыжейке тонкой кишки.

\section{Материал и методы}

В основу работы положены результаты КТ-исследований абдоминальной области
29 пациентов с жиросодержащими образованиями в брыжейке тонкой кишки (16 женщин и 13 мужчин в возрасте от 32 до 81 года, средний возраст 56 лет), проходивших обследование и лечение в ФГБУ «НМИЦ онкологии им. Н.Н. Блохина» Минздрава России. Все обследованные пациенты подписали информированное согласие.

Мезентериальный панникулит отмечался в 13 (45\%) случаях, липосаркомы - в 10 (35\%), у 4 (14\%) пациентов было выявлено лимфопролиферативное заболевание с поражением брыжеечных лимфатических узлов, у 1 (3\%) - лимфоидная гиперплазия смешанного типа, или болезнь Кастлемана, и еще у 1 (3\%) - липома. Морфологические исследования - послеоперационная гистология или пункционная биопсия были выполнены у всех пациентов.

КТ-исследования абдоминальной области проводились на мультиспиральных компьютерных томографах Emotion 6 и Somatom Scope 16 (Siemens). Сначала проводили бесконтрастное (нативное) спиральное сканирование с толщиной среза 5 мм, шагом стола 5 мм, индексом реконструкции 3 мм. Затем, после внутривенного введения рентгеноконтрастного препарата с помощью автоматического инъектора, выполняли серию повторных сканирований в артериальную (при задержке 15-20 с) и венозную (при задержке 40-45 с) фазу, в случае необходимости оценивалась и отсроченная фаза (спустя 5 минут).

\section{Результаты}

По данным КТ-исследования мы оценивали следующие параметры: контуры образования, размеры, структуру (наличие септ и мягкотканных узлов, кальцинатов), взаимосвязь с магистральными сосудами - брыжеечными сосудами, аортой и нижней полой веной (табл. 1). Как следует из таблицы, основной контингент больных с первичной неорганной липосаркомой составляли пожилые люди в возрасте от 54 до 67 лет, тогда как мезентериальный панникулит чаще встречался у людей среднего и пожилого возраста - от 40 лет до 81 года. Лимфопролиферативное заболевание наблюдалось у пациентов от 37 до 73 лет, а забрюшинная липома и болезнь Кастлемана - в более молодом возрасте (в 32 года и 37 лет).

Среди пациентов с первичными неорганными липосаркомами преобладали женщины (3:1). Соотношение полов среди пациентов с мезентериальным панникулитом и лимфопролиферативным поражением было практически равным. 
Таблица 1. Основные демографические характеристики и результаты компьютерной томографической визуализации жиросодержащих абдоминальных новообразований

\begin{tabular}{|c|c|c|c|c|c|c|c|c|}
\hline № $\Pi / \Pi$ & Возраст, годы & Пол & $\begin{array}{r}\text { Морфологическая } \\
\text { принадлежность }\end{array}$ & $\begin{array}{c}\text { Максимальный } \\
\text { размер, см }\end{array}$ & Контуры & $\begin{array}{l}\text { Вовлечение } \\
\text { магистральных } \\
\text { сосудов }\end{array}$ & Кальцинация & $\begin{array}{l}\text { Наличие } \\
\text { мягкотканных } \\
\text { узлов }\end{array}$ \\
\hline 1 & 65 & ж & ЛС & 12 & Четкие & Распластаны & Нет & Нет \\
\hline 2 & 53 & ж & ЛС & 17 & Нечеткие & Распластаны & Нет & Да \\
\hline 3 & 65 & ж & ЛС & 25 & Нечеткие & Распластаны & Нет & Да \\
\hline 4 & 67 & ж & ЛС & 32 & Нечеткие & В структуре & Нет & Да \\
\hline 5 & 59 & $M$ & ЛС & 20 & Нечеткие & Распластаны & Да & Да \\
\hline 6 & 53 & $M$ & ЛС & 35 & Нечеткие & Распластаны & Нет & Да \\
\hline 7 & 60 & M & ЛС & 27 & Нечеткие & Распластаны & Нет & Да \\
\hline 8 & 63 & ж & ЛС & 13 & Нечеткие & Распластаны & Нет & Нет \\
\hline 9 & 58 & ж & ЛС & 5 & Нечеткие & Распластаны & Нет & Нет \\
\hline 10 & 54 & ж & ЛС & 24 & Нечеткие & Распластаны & Нет & Да \\
\hline 11 & 61 & M & МП & 15 & Нечеткие & В структуре & Нет & Да \\
\hline 12 & 61 & $M$ & МП & 9 & Нечеткие & В структуре & Нет & Да \\
\hline 13 & 40 & ж & МП & 9 & Нечеткие & В структуре & Нет & Да \\
\hline 14 & 40 & ж & МП & 13 & Нечеткие & В структуре & Нет & Да \\
\hline 15 & 57 & ж & МП & 14 & Нечеткие & В структуре & Нет & Да \\
\hline 16 & 65 & M & МП & 9 & Нечеткие & В структуре & Нет & Да \\
\hline 17 & 54 & ж & МП & 13 & Нечеткие & В структуре & Нет & Да \\
\hline 18 & 64 & ж & МП & 11 & Нечеткие & В структуре & Нет & Да \\
\hline 19 & 57 & $M$ & МП & 11 & Нечеткие & В структуре & Нет & Да \\
\hline 20 & 51 & ж & МП & 11 & Нечеткие & В структуре & Нет & Да \\
\hline 21 & 44 & $M$ & МП & 11 & Нечеткие & В структуре & Нет & Да \\
\hline 22 & 81 & $M$ & МП & 12 & Нечеткие & В структуре & Нет & Да \\
\hline 23 & 69 & M & МП & 12 & Нечеткие & В структуре & Нет & Да \\
\hline 24 & 73 & ж & Лимфома & 12 & Нечеткие & В структуре & Нет & Да \\
\hline 25 & 59 & M & Лимфома & 9 & Нечеткие & В структуре & Нет & Да \\
\hline 26 & 39 & ж & Лимфома & 10 & Нечеткие & В структуре & Нет & Да \\
\hline 27 & 37 & M & Лимфома & 10 & Нечеткие & В структуре & Нет & Да \\
\hline 28 & 37 & ж & Липома & 12 & Четкие & Распластаны & Да & Да \\
\hline 29 & 32 & $M$ & Болезнь Кастлемана & 12 & Нечеткие & Распластаны & Да & Да \\
\hline
\end{tabular}

ж - женщины, ЛС - липосаркома, м - мужчины, МП - мезентериальный панникулит 


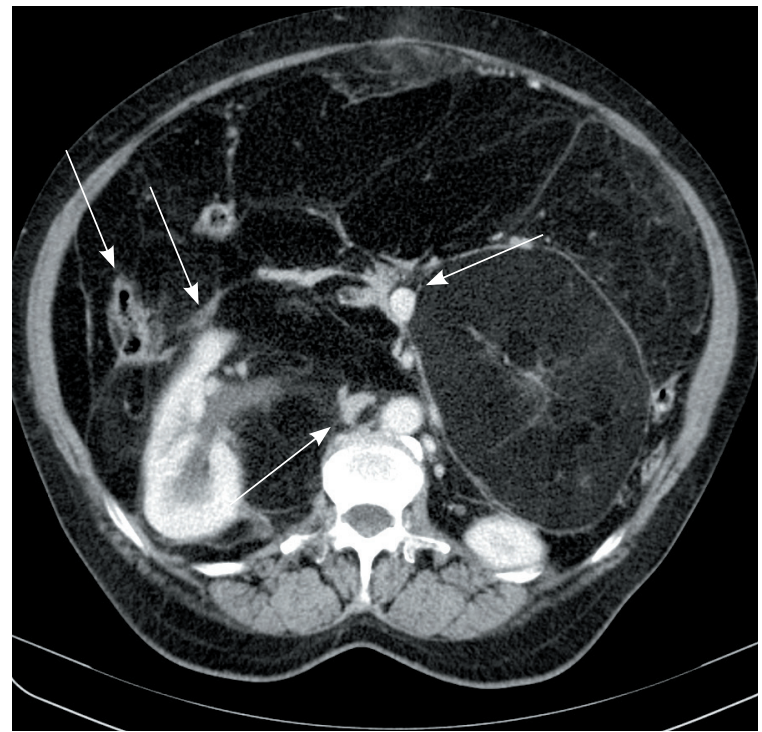

Рис. 1. Пациентка 62 лет с абдоминальной липосаркомой. Аксиальные компьютерные томограммы абдоминальной области (венозная фаза). Всю брюшную полость занимает массивное многоузловое образование жировой структуры, которое окружает правую почку, поджелудочную железу, восходящую ободочную кишку, верхнебрыжеечные сосуды, аорту и нижнюю полую вену (стрелки)

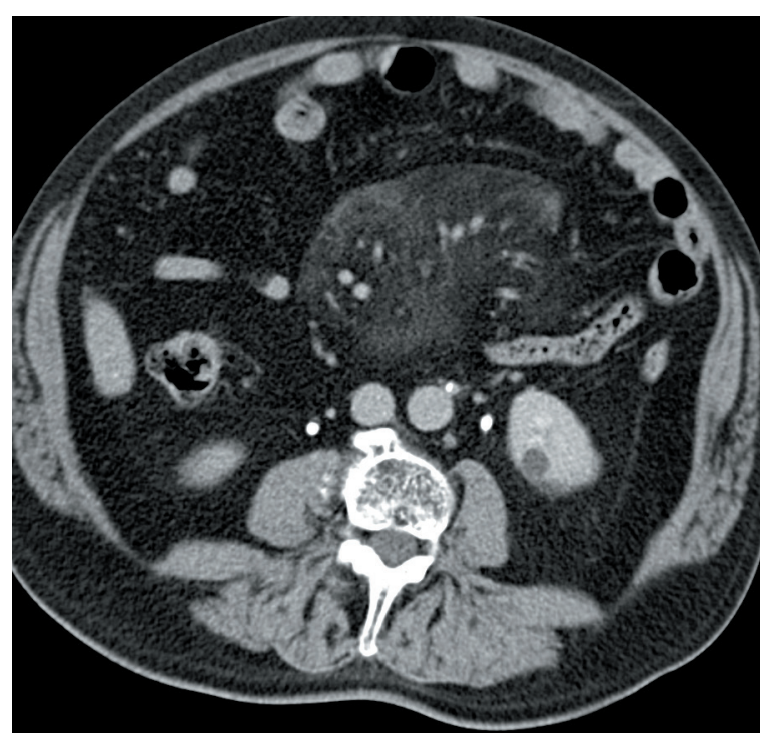

Рис. 3. Пациент 47 лет с мезентериальным панникулитом. Аксиальные компьютерные томограммы абдоминальной области (венозная фаза). В проекции корня брыжейки определяется слабоинтенсивное уплотнение жировой клетчатки в виде объемного образования с частично четкими, частично нечеткими контурами, В структуре которого проходят ветви верхнебрыжеечных сосудов

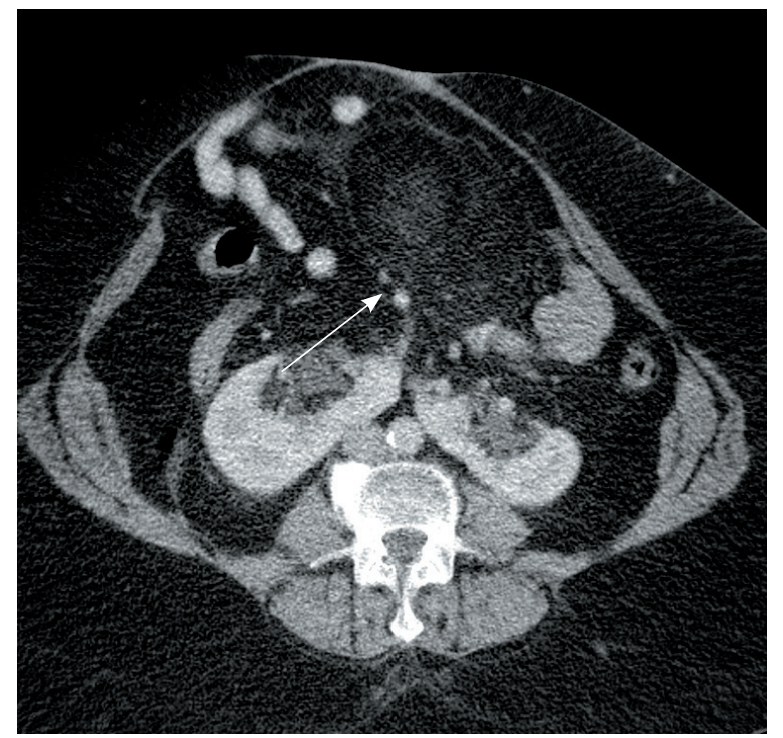

Рис. 2. Пациентка 64 лет с абдоминальной липосаркомой. Аксиальные компьютерные томограммы абдоминальной области (венозная фаза). В брыжейке тонкой кишки определяется опухолевое образование с нечеткими контурами достаточно однородной жировой структуры. Опухоль оттесняет петли тонкой кишки в сторону, по правому контуру распластаны ветви верхнебрыжеечных сосудов (стрелка)

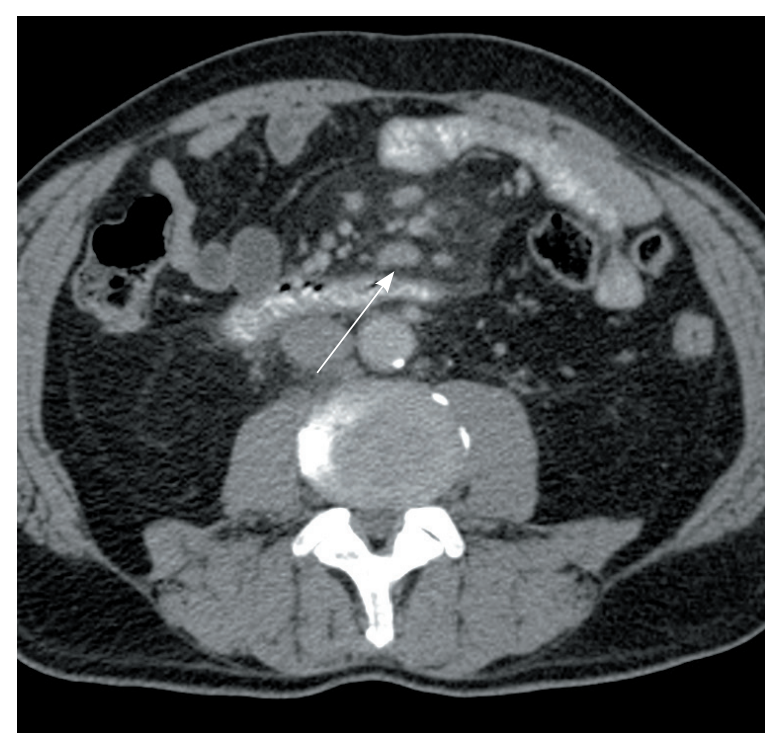

Рис. 4. Пациентка 51 года с мезентериальным панникулитом. Аксиальные компьютерные томограммы абдоминальной области (венозная фаза). В брыжейке тонкой кишки определяется выраженное уплотнение жировой клетчатки в виде объемного образования с нечеткими контурами, неоднородной внутренней структурой за счет множественных увеличенных лимфатических узлов плоской формы (стрелка). Окружающие петли тонкой кишки смещены (раздвинуты) без видимого их вовлечения в процесс 


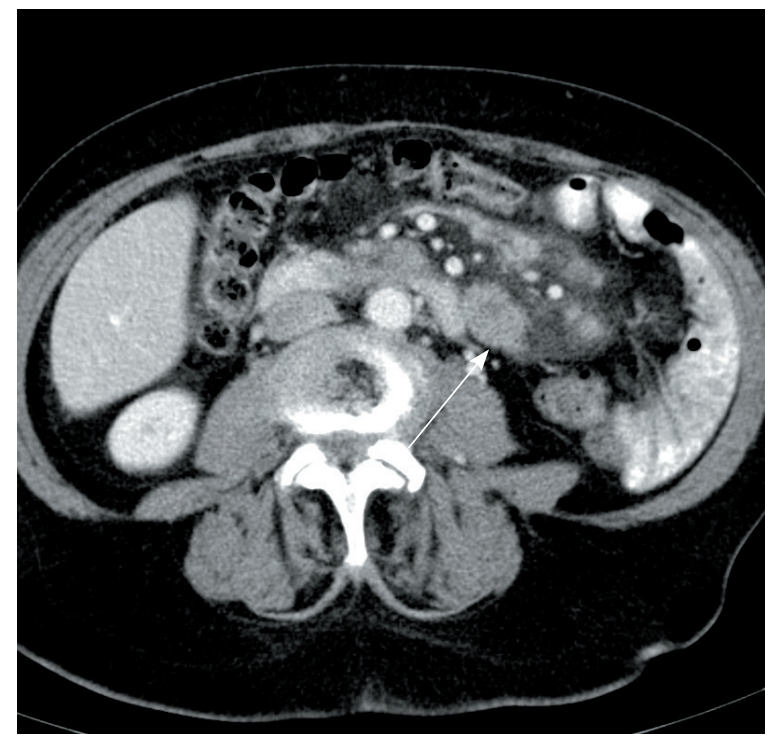

Рис. 5. Пациент 40 лет с лимфомой. Аксиальные компьютерные томограммы абдоминальной области (венозная фаза). Преимущественно в левых отделах брыжейки тонкой кишки определяется выраженное уплотнение жировой клетчатки, в структуре которой визуализируются множественные увеличенные лимфатические узлы с нечеткими контурами, неоднородной структурой за счет участков некроза (стрелка)

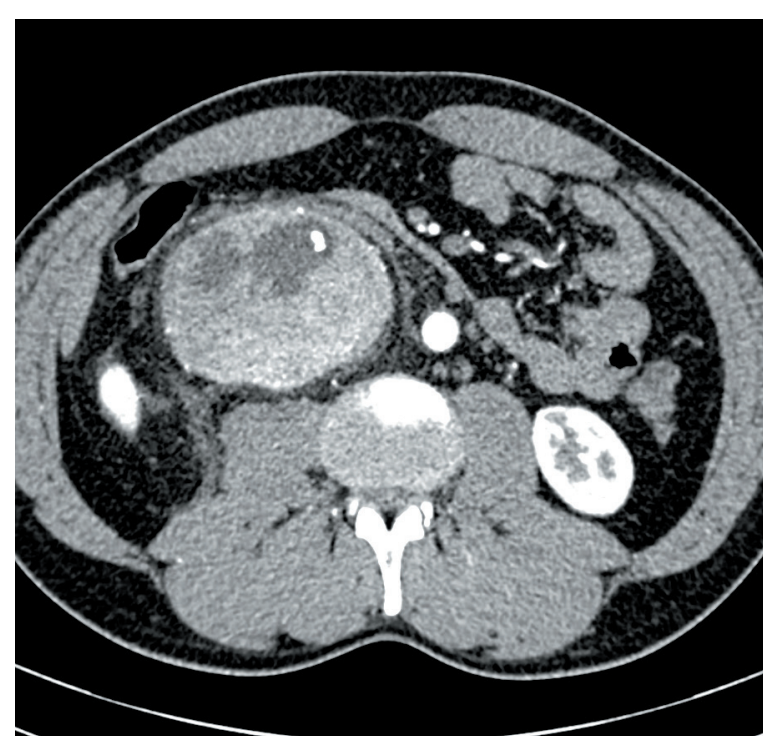

Рис. 7. Пациент 32 лет с болезнью Кастлемана

(ангиофолликулярная гиперплазия лимфатических узлов). Аксиальные компьютерные томограммы абдоминальной области (артериальная фаза). На фоне уплотненной жировой клетчатки позади двенадцатиперстной кишки определяется гиперваскулярное опухолевое образование с четкими контурами, неоднородной внутренней структурой за счет участков пониженной плотности (некроза) и мелких кальцинатов. По переднему контуру опухоли распластана нижняя полая вена (просвет ее сдавлен), петли тонкой и толстой кишки

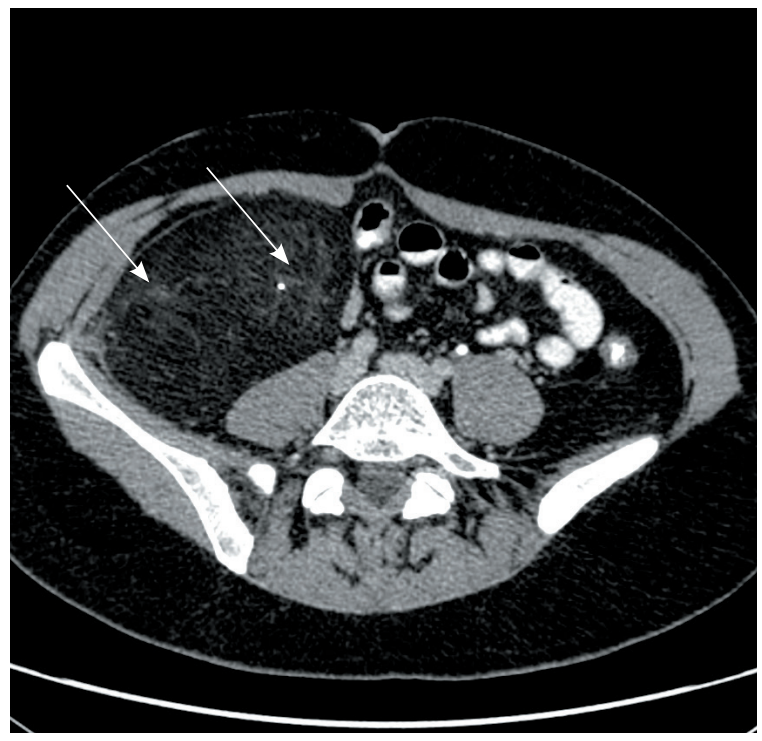

Рис. 6. Пациентка 37 лет с липомой. Аксиальные компьютерные томограммы абдоминальной области (венозная фаза). В правой гипогастральной области с распространением в подвздошную область определяется объемное образование с четкими контурами достаточно однородной жировой структуры с наличием небольшой тяжистости и единичного мелкого кальцината (стрелки). Опухоль смещает петли тонкой кишки влево

Единичный случай липомы зарегистрирован у молодой девушки, а болезнь Кастлемана - у молодого мужчины.

У пациентов с липосаркомой максимальные размеры опухоли достигали 35 см (рис. 1). В большинстве случаев контуры опухоли были нечеткие, и лишь у одного пациента четкие. Внутренняя структура опухоли у всех пациентов представлялась неоднородной за счет наличия мягкотканных узлов (7 из 10) или уплотненных тяжей (3 из 10), в одном случае в структуре опухоли был выявлен мелкий единичный кальцинат размерами до 1 см. В 9 из 10 наблюдений брыжеечные сосуды были оттеснены опухолью в стороны без убедительных признаков их вовлечения в процесс (рис. 2). У одного пациента сосуды проходили в структуре опухоли, просвет их был сужен и деформирован.

При мезентериальном панникулите и лимфопролиферативном поражении максимальные размеры поражения были практически одинаковыми - от 9 до 15 см. Во всех наблюдениях отмечались нечеткие контуры и неоднородная структура, в основном соответствующая уплотненной жировой ткани, на фоне которой при мезентериальном панникулите определялись рассеянные плоские лимфатические узлы (рис. 3, 4), а при 
лимфоме - узловые образования неправильной формы, представляющие собой конгломераты увеличенных лимфатических узлов (рис. 5). Отметим, что у всех пациентов ветви брыжеечных сосудов проходили в толще образования, при лимфоме просвет их был частично сдавлен и деформирован.

У пациентов с липомой и болезнью Кастлемана размеры новообразований были одинаковыми по 12 см. Липома имела четкие контуры и сравнительно однородную жировую структуру с тонкими тяжами и мелким кальцинатом (рис. 6), а при болезни Кастлемана в структуре определялся массивный гиперваскулярный узловой компонент и рассеянные кальцинаты (рис. 7). В обоих случаях ветви брыжеечных сосудов были распластаны в стороны опухолевым образованием.

При анализе отдельных мягкотканных узлов в толще образования мы пользовались разработанным нами перечнем основных КТпроявлений, приведенных в табл. 2. Среди доброкачественных жиросодержащих образований в области брыжейки тонкой кишки в наших наблюдениях наиболее часто встречался мезентериальный панникулит. В большинстве случаев в его структуре определялись множественные (7 из 13, 53,8\%) и немногочисленные (5 из $13,38,5 \%)$ узловые образования овальной формы с размерами от 0,7 до 2 см (12 из 13, 92,3\%), в единичном наблюдении - до 4 см. Практически все мягкотканные узлы имели четкие контуры и сравнительно однородную внутреннюю структуру. После введения контрастного препарата отмечалась слабая (7 из 13, 53,8\%) и умеренная (6 из 13, 46,1\%) однородная степень усиления узлов в венозную фазу исследования.

У 7 из 10 пациентов с липосаркомой были обнаружены мягкотканные включения в толще жировой опухоли. Во всех наших наблюдениях количество узлов всегда представлялось разным, от единичных до множественных (неисчисляемых), размерами от 0,5 до 10 см. В отличие от мезентериального панникулита, узлы при липосаркоме имели неправильную форму (5 из 7, 71,4\%) и нечеткие тяжистые контуры. Внутренняя структура мягкотканного компонента в равной степени была однородной или неоднородной за счет участков пониженной плотности (некроза). После введения контрастного средства наблюдали слабое или умеренное диффузное гетерогенное усиление узлов преимущественно в отсроченную фазу исследования.

При лимфопролиферативном заболевании регистрировали множественные узловые образования различных размеров (от 1 до $5 \mathrm{~cm}$ ) с тенденцией к слиянию в массивные конгломераты. У всех узлов была неправильная форма, четкие контуры и неоднородная внутренняя структура. При болюсном внутривенном контрастировании опухолевые ткани выраженно накапливали контрастный препарат в артериальную фазу исследования (3 из 4, 75\%).

Болезнь Кастлемана, или лимфоидная гиперплазия смешанного типа, встречается редко. Подобно липосаркомам, в нашем единичном наблюдении новообразование имело преимущественно солидную неоднородную структуру, неправильную форму, четкие контуры и крупные размеры (до 8 см), но в отличие от всех липосарком характеризовалось диффузным неоднородным контрастированием в артериальную фазу исследования. Следует отметить, что дифференциальная диагностика забрюшинной липосаркомы и лимфоидной гиперплазии смешанного типа была весьма сложной задачей, и в конкретном случае возникла необходимость в проведении морфологической верификации.

Результаты анализа заключений рентгеновской КТ (РКТ) с внутривенным болюсным контрастированием показали, что липосаркома была диагностирована в 12 случаях, мезентериальный панникулит - в 13 и лимфома в 4. После морфологической верификации всех 29 случаев диагноз липосаркомы подтвердился в 10 случаях из 12 заключений РКТ, у остальных 2 пациентов диагноз оказался неверным (у 1 пациента была липома, у другого - болезнь Кастлемана). Мезентериальный панникулит и лимфопролиферативное заболевание при РКТ были диагностированы правильно.

В своей работе мы использовали общеизвестные определения эффективности диагностического исследования [14]. Истинноположительный (ИП) результат - положительные результаты у людей, действительно больных данной болезнью. В нашем исследовании ИП результат при РКТ выявлен в 27 из 29 случаев. Ложноотрицательные (ЛО) результаты - отрицательные результаты у больных людей. В нашей работе ЛО результат при РКТ выявлен в 2 из 29 случаев. Чувствительность - способность диагностического метода давать правильный результат, что определяется как доля истинноположительных результатов среди проведенных тестов (Чувствительность $=$ ИП / (ИП + ЛО $) \times 100 \%)$. Таким образом, чувствительность РКТ с внутривенным болюсным контрастированием в выявлении жиросодержащих новообразований в брюшной полости составила $93,1 \%$. 
Таблица 2. Семиотические компьютерно-томографические признаки мягкотканных узлов в структуре жиросодержащих новообразований

\begin{tabular}{|c|c|c|c|c|c|c|}
\hline \multirow[t]{2}{*}{ № $п / п$} & \multirow[t]{2}{*}{ КТ-проявление } & \multicolumn{5}{|c|}{ Результаты, абс. (\%) } \\
\hline & & $Л C, n=7$ & $\mathrm{M} \Pi, \mathrm{n}=13$ & лимфома, $\mathrm{n}=4$ & липома, $\mathrm{n}=1$ & $\mathrm{EK}, \mathrm{n}=1$ \\
\hline \multirow[t]{4}{*}{1} & Количество узлов & & & & & \\
\hline & единичные (1-2) & $3(42,9)$ & $1(7,7)$ & - & - & $1(100)$ \\
\hline & немногочисленные (3-4) & $2(28,6)$ & $5(38,5)$ & - & - & - \\
\hline & множественные (более 4) & $2(28,6)$ & $7(53,8)$ & $4(100)$ & - & - \\
\hline \multirow[t]{5}{*}{2} & Размеры узлов, см & & & & & \\
\hline & $1-2$ & $2(28,6)$ & $12(92,3)$ & $1(25)$ & - & - \\
\hline & $2-4$ & $2(28,6)$ & $1(7,7)$ & $2(50)$ & - & - \\
\hline & $4-8$ & - & - & $1(25)$ & - & - \\
\hline & более 8 & $3(42,9)$ & - & - & - & $1(100)$ \\
\hline \multirow[t]{4}{*}{3} & Форма узлов & & & & & \\
\hline & овальная & $1(14,3)$ & $12(92,3)$ & - & - & - \\
\hline & круглая & $1(14,3)$ & $1(7,7)$ & - & - & - \\
\hline & неправильная & $5(71,4)$ & - & $4(100)$ & - & $1(100)$ \\
\hline \multirow[t]{3}{*}{4} & Контуры узлов & & & & & \\
\hline & четкие & $2(28,6)$ & $13(100)$ & $4(100)$ & - & - \\
\hline & нечеткие & $5(71,4)$ & - & - & - & $1(100)$ \\
\hline \multirow[t]{3}{*}{5} & Структура узлов & & & & & \\
\hline & однородная & $3(42,9)$ & $13(100)$ & - & - & - \\
\hline & неоднородная & $4(57,1)$ & - & $4(100)$ & - & $1(100)$ \\
\hline \multirow[t]{4}{*}{6} & Степень контрастирования узлов & & & & & \\
\hline & слабая & $3(42,9)$ & $7(53,8)$ & - & - & - \\
\hline & умеренная & $4(57,1)$ & $6(46,1)$ & $1(25)$ & - & - \\
\hline & выраженная & - & - & $3(75)$ & - & $1(100)$ \\
\hline \multirow[t]{4}{*}{7} & Максимальное контрастирование узлов & & & & & \\
\hline & артериальная фаза & - & - & $4(100)$ & - & $1(100)$ \\
\hline & венозная фаза & - & $12(92,3)$ & - & - & - \\
\hline & отсроченная фаза & $7(100)$ & $1(7,7)$ & - & - & - \\
\hline
\end{tabular}




\section{Обсуждение}

Липома - доброкачественная опухоль, состоящая из зрелой жировой ткани [15]. Липома в абдоминальной области выступает крайне редкой находкой и встречается чаще у женщин в различных возрастных группах $[8,16,17]$. Микроскопически опухоль состоит из зрелых адипоцитов, идентичных окружающей их жировой ткани с небольшими вариациями размеров и формы клеток [8]. Размеры опухоли в среднем составляют 1-5 см, однако в литературе описаны случаи, когда опухоль достигала гигантских объемов $[17,18]$.

Обычно липома имеет округлую или овальную форму, четкие и ровные контуры, капсулу, достаточно однородную структуру и плотность, аналогичную жировой ткани. В структуре липомы могут визуализироваться единичные тонкие перегородки, и в редких случаях - мягкотканный компонент. После внутривенного усиления накопления контрастного препарата в опухоли не происходит [9]. В нашем наблюдении в структуре липомы визуализировались множественные септы и мелкий кальцинат. Согласно данным литературы, кальцификация может присутствовать в 11\% случаев, хотя чаще всего ассоциируется с высокодифференцированной липосаркомой [19]. Учитывая низкую частоту встречаемости и вышеперечисленные признаки, ее практически невозможно отличить от высокодифференцированной липосаркомы.

Высокодифференцированная липосаркома - относительно медленно растущая опухоль, которая представляет собой наиболее часто встречаемый гистологический подтип среди всех липосарком. В большинстве случаев она напоминает липому больших размеров с плотными коллагеновыми структурами [10]. Источником забрюшинных липосарком чаще служит паранефральная клетчатка, реже клетчатка тонкой и толстой кишки $[11,20]$.

Высокодифференцированная липосаркома обычно наблюдается у людей в возрасте от 50 до 70 лет и не зависит от половой принадлежности [21], однако в нашем исследовании преобладали женщины. Как правило, липосаркомы протекают бессимптомно и становятся случайной находкой, когда опухоль достигает значительных размеров и начинает воздействовать на близлежащие структуры. Высокодифференцированная липосаркома имеет низкую степень злокачественности и не обладает метастатическим потенциалом. По мнению ряда авторов, она может перерождаться в дедифференцированную липосаркому, что непосредственно влияет на частоту местных рецидивов и прогноз заболевания [22-24].

Высокодифференцированные липосаркомы можно разделить на три подтипа: адипоцитарные (или липомоподобные), склерозирующие и воспалительные. Липомоподобный подтип представляет собой жировую опухоль с более толстыми и неправильными или узловатыми перегородками, чем те, которые наблюдаются в липомах [12]. Склерозирующий подтип демонстрирует наличие более плотного компонента в структуре, который приближается к характеристикам мышцы. Воспалительный подтип, который встречается относительно редко и имеет гистологическую особенность обширной лимфоплазмоцитарной инфильтрации, проявляется на КТ в виде фиброзно-жировой массы [25]. По данным W.D. Craig и соавт., наличие кальцинатов наблюдается в 30\% случаев и служит важным признаком дедифференцировки [11].

В большинстве наблюдений при РКТ липосаркома определяется в виде опухоли крупных размеров - более 20 см (в 1,5\% случаев - менее 5 см), с менее четкими контурами, чем у липомы [26]. Основная масса представлена жировой тканью с наличием неравномерно утолщенных перегородок или участков высокой плотности, соответствующих участкам фиброза и склероза [27]. Перегородки и мягкотканные компоненты при РКТ с внутривенным контрастированием постепенно и равномерно накапливают контрастный препарат [25, 28].

Роль мягкотканного компонента в структуре высокодифференцированной жировой опухоли изучена мало, его детальная характеристика в мировой литературе приводится лишь в единичных научных работах [29, 30]. Наши результаты показали, что количество и размеры узловых образований в структуре высокодифференцированной липосаркомы могут быть различными, а контуры - нечеткими. Внутренняя структура узлов в равной степени может быть однородной или неоднородной за счет участков некроза и фиброза, максимальное контрастирование которых приходится на отсроченную фазу исследования.

Мезентериальный панникулит - редкое идиопатическое заболевание, характеризующееся хроническим неспецифическим воспалением с вовлечением жировой ткани брыжейки кишки, а также сальника, предбрюшинной и забрюшинной клетчатки $[5,31]$. Он наблюдается у людей среднего и пожилого возраста, в равной степени 
у мужчин и женщин [31,32]. При мезентериальном панникулите могут встречаться такие клинические симптомы, как боль в животе, рвота, потеря веса, нарушение работы кишечника, реже - лихорадка неизвестного происхождения, кровотечения и желтуха [32, 33]. У большинства пациентов заболевание протекает бессимптомно и служит случайной находкой при инструментальных методах исследования. Следует обратить внимание на то, что описанные клинические проявления не встречаются при лимфопролиферативном заболевании, липосаркомах и липомах [13].

Визуально мезентериальный панникулит при РКТ можно разделить на диффузный и узловой типы поражения. Диффузный тип поражения проявляется как слабоинтенсивное уплотнение жировой ткани вдоль брыжеечных сосудов, при узловом типе отмечается наличие мягкотканного образования в области корня брыжейки. Мягкотканные участки представляют собой элементы воспалительной инфильтрации, фиброза и отека [34], возможно наличие кальцинатов [5].

По данным литературы, максимальные размеры патологического образования варьируют в пределах от 7 до 15 см. Мезентериальный панникулит окружает брыжеечные сосуды без сужения их просвета и может смещать смежные петли тонкой кишки без признаков инвазии. Кроме того, могут быть обнаружены псевдокапсула толщиной от 2 до 8 мм и увеличенные лимфатические узлы в брыжейке, реже в забрюшинной области. Вокруг узлов и сосудов часто определяется жировой ободок (симптом «жирового кольца», англ. fat ring). Узлы, как правило, имеют четкие контуры и не накапливают контрастный препарат [31, 35-38].

Первичная лимфома с изолированным поражением мезентериальных лимфатических узлов встречается крайне редко и часто ассоциирована с неходжкинской лимфомой [34]. Обычно встречается у людей старше 60 лет, тогда как болезнь Ходжкина наблюдается в молодом и среднем возрасте. Кроме того, более восприимчивы к лимфопролиферативному поражению больные СПИДом [39, 40]. Мезентериальные лимфомы могут достигать больших размеров, не вызывая клинических симптомов [41].

R.S. Yu и соавт. в своем исследовании выделили следующие типы поражения лимфатических узлов при РКТ: солитарный, многоузловой и диффузный. Солитарный тип характеризуется наличием единичных лимфатических узлов с тенденцией к слиянию, округлой формы, однородной структуры, размерами до 3-6 см в диаметре. Многоузловой тип наблюдается в виде массивного конгломерата в области корня брыжейки размерами от 8 до 18 см, с четкими контурами, однородной или неоднородной внутренней структурой. Диффузный тип проявляется наличием множественных, не сливающихся между собой лимфатических узлов в брыжейке тонкой кишки и забрюшинной области (ниже уровня 2-3-го поясничных позвонков) [42-44].

После внутривенного контрастирования отмечается незначительное или умеренное контрастирование узлов, редко - усиленное. Для лимфопролиферативного поражения характерно окружение и сдавление брыжеечных сосудов, брюшной аорты и нижней полой вены, а также деформации просвета тонкой кишки $[44,45]$.

В нашем исследовании у всех лимфатических узлов была неоднородная внутренняя структура и выраженное накопление контрастного препарата в артериальную фазу исследования.

Болезнь Кастлемана (ангиофолликулярная гиперплазия лимфатических узлов) - редкое доброкачественное лимфопролиферативное заболевание с длительным бессимптомным течением неизвестной этиологии. Учитывая низкую частоту встречаемости и единичные публикации в отечественной литературе, считаем целесообразным осветить особенности данного заболевания более подробно. Впервые патология была описана B. Castleman в 1954 г. как пролиферация зрелых лимфоцитов и/или плазматических клеток [46]. Болезнь Кастлемана регистрируют во всех возрастных группах (70\% пациентов обычно моложе 35 лет, средний возраст 40 лет), одинаково часто у мужчин и женщин [47]. Заболевание можно разделить на две формы: одиночную (наиболее распространенная) и мультицентрическую. Последняя форма характеризуется диссеминированной лимфаденопатией и всегда связана с системными симптомами, такими как лихорадка, гематологические нарушения, включая анемию и гипергаммаглобулинемию, эндокринологические нарушения и периферические невропатии [48-50]. В 70\% случаев выявляется единичное образование в средостении, крайне редко - в других областях, в том числе в брыжейке кишки $[51,52]$.

При РКТ обычно определяется узловое образование мягкотканной структуры с усилением от умеренного до интенсивного [53, 54]. Кальцификация встречается в $50 \%$ случаев, особенно часто при расположении в абдоминальной области или области таза $[55,56]$. В исследовании T.L. Meador и J.K. McLarney размеры 
новообразования варьировались от 1 до $18 \mathrm{~cm}$, при этом опухоль диаметром менее 5 см обычно имела однородную структуру, тогда как при диаметре более 5 см демонстрировала гетерогенную структуру за счет участков некроза; во всех случаях было выявлено усиленное контрастирование [52]. В нашем наблюдении вокруг мягкотканного узлового образования определялась выраженно уплотненная жировая клетчатка, что имитировало липосаркому.

Редкая встречаемость данного заболевания и схожая рентгенологическая картина с другими доброкачественными и злокачественными мезентериальными образованиями вызывают диагностические трудности, что определяет необходимость дальнейшей морфологической верификации. По данным литературы, отличительными признаками болезни Кастлемана от лимфом выступают отсутствие кальцинации и умеренное накопление контрастного препарата, однако в нашем наблюдении в обоих случаях

\section{Дополнительная информация}

\section{Финансирование}

Работа проведена без привлечения дополнительного финансирования со стороны третьих лиц.

\section{Конфликт интересов}

Авторы декларируют отсутствие явных и потенциальных конфликтов интересов, связанных с публикацией настоящей статьи. отмечалось одинаковое контрастирование, выраженное в артериальную фазу исследования. Забрюшинные липосаркомы, как правило, накапливают контрастный препарат в отсроченную фазу и могут иметь в своей структуре кальцинаты в зависимости от гистологического типа.

\section{Заключение}

Выявление и оценка структуры жиросодержащих образований в области брыжейки тонкой кишки служат основной задачей при анализе РКТизображений. Полученные данные в большинстве случаев имеют решающее значение для дифференциальной диагностики между поражениями, при которых необходимо хирургическое вмешательство, и теми, при которых показано медикаментозное лечение или вообще не нужно лечения. В ряде случаев при редко встречающихся в клинической практике образованиях, таких как болезнь Кастлемана, требуется проведение морфологической верификации. (६)

\section{Участие авторов}

Оба автора внесли равный вклад в проведение исследования и подготовку статьи, прочли и одобрили финальную версию статьи перед публикацией, согласны нести ответственность за все аспекты работы и гарантируют, что ими надлежащим образом были рассмотрены и решены вопросы, связанные с точностью и добросовестностью всех частей работы.

\section{Литература / References}

1.Liles JS, Tzeng CW, Short JJ, Kulesza P, Heslin MJ. Retroperitoneal and intra-abdominal sarcoma. Curr Probl Surg. 2009;46(6):445-503. doi: 10.1067/j.cpsurg.2009.01.004.

2. Dalal KM, Kattan MW, Antonescu CR, Brennan MF, Singer S. Subtype specific prognostic nomogram for patients with primary liposarcoma of the retroperitoneum, extremity, or trunk. Ann Surg. 2006;244(3):381-391. doi: 10.1097/01.sla.0000234795.98607.00.

3. Kindblom LG, Angervall L, Svendsen P. Liposarcoma a clinicopathologic, radiographic and prognostic study. Acta Pathol Microbiol Scand Suppl. 1975;(253):1-71.

4. Fletcher CDM, Bridge JA, Hogendoorn PCW, Mertens $\mathrm{F}$, editors. WHO classification of tumours of soft tissue and bone. $4^{\text {th }}$ ed. WHO Press; 2013.

5.Лукьянченко АБ, Долгушин БИ, Стилиди ИС, Медведева БМ. Мезентериальный панникулит (обзор литературы и собственные наблюдения). Медицинская визуализация. 2005;(4):70-75. [Lukjanchenko AB,
Dolgushin BI, Stilidi IS, Medvedeva BM. [Mesenteric panniculitis (the review and own experience)]. Medical Visualization. 2005;(4):70-75. Russian.]

6. Nishino M, Hayakawa K, Minami M, Yamamoto A, Ueda H, Takasu K. Primary retroperitoneal neoplasms: $C T$ and $M R$ imaging findings with anatomic and pathologic diagnostic clues. Radiographics. 2003;23(1):45-57. doi: 10.1148/ rg.231025037. Erratum in: Radiographics. 2003;23(5):1340.

7. Scali EP, Chandler TM, Heffernan EJ, Coyle J, Harris AC, Chang SD. Primary retroperitoneal masses: what is the differential diagnosis? Abdom Imaging. 2015;40(6):1887-1903. doi: 10.1007/s00261-014-0311-x.

8. Nielsen GP, Mandahl N. Lipoma. In: Fletcher CDM, Unni KK, Mertens F, editors. World Health Organization Classification of Tumours. Pathology and Genetics of Tumours of Soft Tissue and Bone. Lyon: IARC Press; 2002. p. 20-22.

9. Vlasov PV, Kotliarov PM. Complex radiation diagnosis of retroperitoneal tumors and tumor-like states. Vestn Rentgenol Radiol. 1998;(3):30-40.

10. Weiss SW. Lipomatous tumors. In: Weiss SW, Brooks JSJ, editors. Soft tissue tumors. Baltimore, Md: Williams \& Wilkins; 1996. p. 207-251.

11. Craig WD, Fanburg-Smith JC, Henry LR, Guerrero $\mathrm{R}$, Barton JH. Fat-containing lesions of the retroperitoneum: radiologic-pathologic correlation. Radiographics. 2009;29(1):261-290. doi: 10.1148/rg.291085203.

12. Pereira JM, Sirlin CB, Pinto PS, Casola G. CT and $M R$ imaging of extrahepatic fatty masses of the abdomen and pelvis: techniques, diagnosis, differential diagnosis, and pitfalls. Radiographics. 2005;25(1):69-85. doi: 10.1148/ rg. 251045074 .

13. Friedman AC, Hartman DS, Sherman J, Lautin EM, Goldman M. Computed tomography of abdominal fatty masses. Radiology. 1981;139(2):415-429. doi: 10.1148/radiology.139.2.7220888.

14. Кармазановский ГГ. Оценка диагностической значимости метода («чувстви- 
тельность», «специфичность», «общая точность»). Анналы хирургической гепатологии. 1997;2:139-142. [Karmazanovskiy GG. [Evaluation of the diagnostic significance of the method ("sensitivity", "specificity", "general accuracy")]. Annals of HPB Surgery. 1997;2:139-142. Russian.]

15. Weniger M, D'Haese JG, Kunz W, Pratschke S, Guba M, Werner J, Angele MK. En-bloc resection of a giant retroperitoneal lipoma: a case report and review of the literature. BMC Res Notes. 2015;8:75. doi: 10.1186/s13104-0151038-7.

16. Ukita S, Koshiyama M, Ohnaka M, Miyagawa N, Yamanishi $Y$, Nishimura F, Nagura M, Kim T, Hirose $M$, Shirase $T$, Kobayashi $H$, Ozasa $H$. Retroperitoneal lipoma arising from the urinary bladder. Rare Tumors. 2009;1(1):e13. doi: 10.4081/rt.2009.e13.

17. Al-Ali MHM, Salih AM, Ahmed OF, Kakamad FH, Mohammed SH, Hassan MN, Sidiq SH, Mustafa MQ, Najar KA, Abdullah IY. Retroperitoneal lipoma; a benign condition with frightening presentation. Int J Surg Case Rep. 2019;57:6366. doi: 10.1016/j.ijscr.2019.02.044.

18. Королева ИМ, Кондрашин СА. Гигантская липома брюшной полости. Российский электронный журнал лучевой диагностики. 2012;2(1):76-79. [Koroleva IM, Kondrashin SA. [Giant abdominal lipoma]. Russian Electronic Journal of Radiology. 2012;2(1):76-79. Russian.]

19. Murphey MD, Carroll JF, Flemming DJ, Pope TL, Gannon FH, Kransdorf MJ. From the archives of the AFIP: benign musculoskeletal lipomatous lesions. Radiographics. 2004;24(5):1433-1466. doi: 10.1148/rg.245045120.

20. Meloni F, Feo CF, Profili S, Cossu ML, Meloni GB. Omental Well-Differentiated Liposarcoma: US, CT and MR Findings. Int J Biomed Sci. 2009;5(3):302-304.

21. Weiss SW, Goldblum JR. Enzinger and Weiss's soft tissue tumors. $5^{\text {th }}$ ed. St Louis, Mo: Mosby; 2007.

22. Lindberg MR. Diagnostic Pathology: Soft Tissue Tumors. $2^{\text {nd }}$ ed. Elsevier; $2015.800 \mathrm{p}$.

23. Kilpatrick SE, Doyon J, Choong PF, Sim FH, Nascimento AG. The clinicopathologic spectrum of myxoid and round cell liposarcoma. A study of 95 cases. Cancer. 1996;77(8): 1450-1458. doi: 10.1002/(SICI)1097$0142(19960415) 77: 8<1450:: A I D-C N$ CR5 > 3.0.CO;2-G.

24. Weiss SW, Rao VK. Well-differentiated liposarcoma (atypical lipoma) of deep soft tissue of the extremities, retroperitoneum, and miscellaneous sites. A follow-up study of 92 cases with analysis of the incidence of "dedifferentiation". Am J Surg Pathol. 1992;16(11):10511058. doi: 10.1097/00000478-19921100000003.

25. Kawano R, Nishie A, Yoshimitsu K, Irie H, Tajima T, Hirakawa M, Ishigami K, Ushijima Y, Oka- moto D, Yabuuchi H, Taketomi A, Nishihara $Y$, Fujita N, Honda H. Retroperitoneal well-differentiated inflammatory liposarcoma: a diagnostic dilemma. Radiat Med. 2008;26(7): 450-453. doi: 10.1007/s11604-008-0255-6.

26. Неред СН, Стилиди ИС, Клименков АА, Болотский ВИ, Анурова ОА. Клинико-морфологические особенности и результаты хирургического лечения забрюшинных неорганных липосарком. Вопросы онкологии. 2012;58(1):94-100. [Nered SN, Stilidi IS, Klimenkov AA, Bolotsky VI, Anurova OA. [Clinico-morphological properties and surgical treatment results in retroperitoneal liposarcomas]. Problems in Oncology. 2012;58(1): 94-100. Russian.]

27. Павлов КА, Дубова ЕА, Щеголев АИ, Мишнев ОД, Кармазановский ГГ, Кунцевич ГИ, Степанова ЮА. Липосаркома забрюшинного пространства. Медицинская визуализация. 2007;(3):72-80. [Pavlov KA, Dubova EA, Shchegolev Al, Mishnjev OD, Karmazanovsky GG, Kuntsevich Gl, Stepanova YuA. [Liposarcoma of retroperitoneal space]. Medical Visualization. 2007;(3):72-80. Russian.]

28. Nishimura $H$, Zhang $Y$, Ohkuma K, Uchida M, Hayabuchi N, Sun S. MR imaging of soft-tissue masses of the extraperitoneal spaces. Radiographics. 2001;21(5):1141-1154. doi: 10.1148/ radiographics.21.5.g01se141141.

29. Bhosale P, Wang J, Varma D, Jensen C, Patnana $M$, Wei W, Chauhan A, Feig B, Patel $S$, Somaiah N, Sagebiel T. Can Abdominal Computed Tomography Imaging Help Accurately Identify a Dedifferentiated Component in a Well-Differentiated Liposarcoma? J Comput Assist Tomogr. 2016;40(6):872-879. doi: 10.1097/RCT.0000000000000462.

30. Kim T, Murakami T, Oi H, Tsuda K, Matsushita M, Tomoda K, Fukuda H, Nakamura H. CT and MR imaging of abdominal liposarcoma. AJR Am J Roentgenol. 1996;166(4):829-833. doi: 10.2214/ajr.166.4.8610559.

31. Daskalogiannaki M, Voloudaki A, Prassopoulos P, Magkanas E, Stefanaki K, Apostolaki E, Gourtsoyiannis N. CT evaluation of mesenteric panniculitis: prevalence and associated diseases. AJR Am J Roentgenol. 2000;174(2): 427-431. doi: 10.2214/ajr.174.2.1740427.

32. Durst AL, Freund H, Rosenmann E, Birnbaum D. Mesenteric panniculitis: review of the leterature and presentation of cases. Surgery. 1977;81(2):203-211.

33. Akram S, Pardi DS, Schaffner JA, Smyrk TC. Sclerosing mesenteritis: clinical features, treatment, and outcome in ninety-two patients. Clin Gastroenterol Hepatol. 2007;5(5):589-596; quiz 523-524. doi: 10.1016/j.cgh.2007.02.032.

34. Anis M, Irshad A. Imaging of abdominal lymphoma. Radiol Clin North Am. 2008;46(2):265285, viii-ix. doi: 10.1016/j.rcl.2008.04.001.

35. Kopecky KK, Lappas JC, Baker MK, Madura JA. Mesenteric panniculitis: CT appearance. Gas- trointest Radiol. 1988;13(3):273-274. doi: 10.1007/BF01889079.

36. Katz ME, Heiken JP, Glazer HS, Lee JK. Intraabdominal panniculitis: clinical, radiographic, and CT features. AJR Am J Roentgenol. 1985;145(2): 293-296. doi: 10.2214/ajr.145.2.293.

37. Sans $M$, Varas $M$, Anglada $A$, Esperanza Bachs $M$, Navarro S, Brugués J. Mesenteric panniculitis presenting as fever of unknown origin. Am J Gastroenterol. 1995;90(7):1159-1161.

38. Lee JKT, Sagel SS, Stanley RJ. Computed Body Tomography with MRI Correlation. $2^{\text {nd }}$ ed. New York: Raven Press; 1988. 1184 p. doi: 10.1002/ mrm.1910110113.

39. Feugier P, Van Hoof A, Sebban C, Solal-Celigny $\mathrm{P}$, Bouabdallah $\mathrm{R}$, Fermé $\mathrm{C}$, Christian B, Lepage E, Tilly $H$, Morschhauser F, Gaulard P, Salles G, Bosly A, Gisselbrecht C, Reyes $F$, Coiffier $B$. Long-term results of the $\mathrm{R}-\mathrm{CHOP}$ study in the treatment of elderly patients with diffuse large B-cell lymphoma: a study by the Groupe d'Etude des Lymphomes de l'Adulte. J Clin Oncol. 2005;23(18): 4117-4126. doi: 10.1200/JCO.2005.09.131.

40. Radin DR, Esplin JA, Levine AM, Ralls PW. AIDS-related non-Hodgkin's lymphoma: abdominal CT findings in 112 patients. AJR Am J Roentgenol. 1993;160(5):1133-1139. doi: 10.2214/ajr.160.5.8470595.

41. Hardy SM. The sandwich sign. Radiology. 2003;226(3):651-652. doi: 10.1148/radiol.2263020109.

42. Yu RS, Zhang WM, Liu YQ. CT diagnosis of 52 patients with lymphoma in abdominal lymph nodes. World J Gastroenterol. 2006;12(48): 7869-7873. doi: 10.3748/wjg.v12.i48.7869.

43. Yang ZG, Min $P Q$, Sone $S$, He ZY, Liao ZY, Zhou XP, Yang GQ, Silverman PM. Tuberculosis versus lymphomas in the abdominal lymph nodes: evaluation with contrast-enhanced $\mathrm{CT}$. AJR Am J Roentgenol. 1999;172(3):619-623. doi: 10.2214/ajr.172.3.10063847.

44. Young ZG, Wen PQ, Liao ZY, He ZY, Zhou XP, Yang HX. CT features and anatomic distribution of malignant lymphoma in abdominal lymph nodes involvement. Zhonghua Fangshe Zazie. 1996;30:727-731.

45. Zhou KR. CT of the Abdomen. $1^{\text {th }}$ ed. Shanghai: Shanghai Medical University publisher; 1993.

46. CASE records of the Massachusetts General Hospital Weekly Clinicopathological Exercises: Case 40011. N Engl J Med. 1954;250(1):26-30. doi: 10.1056/NEJM195401072500107.

47. Casper C. The aetiology and management of Castleman disease at 50 years: translating pathophysiology to patient care. $\mathrm{Br} J \mathrm{Hae}-$ matol. 2005;129(1):3-17. doi: 10.1111/j.13652141.2004.05311.x.

48. Libson E, Fields S, Strauss S, Bloom RA, Okon E, Galun E, Polliack A. Widespread Castleman disease: CT and US findings. Radiology. 1988;166(3):753-755. doi: 10.1148/radiology.166.3.3277245. 
49. Hung IJ, Kuo TT, Lin JN. New observations in a child with angiofollicular lymph node hyperplasia (Castleman's disease) originated from the mesenteric root. Am J Pediatr Hematol Oncol. 1992;14(3):255-260. doi: 10.1097/00043426-199208000-00013.

50. Jean SN, Hwang SJ, Ju S, Jan H, Chylo T, Shu HF. Mesenteric Castleman disease: A Case Report. Chin J Radiol. 2001;26:265-268.

51. Lee JP, Ngai RL. Mesenteric giant lymph node hyperplasia - report of a case and review of the literature. Australas Radiol. 1990;34(2):162164. doi: 10.1111/j.1440-1673.1990.tb02834.x.
52. Meador TL, McLarney JK. CT features of Castleman disease of the abdomen and pelvis. AJR Am J Roentgenol. 2000;175(1):115-118. doi: 10.2214/ajr.175.1.1750115.

53. Malara FA, Price D, Fabiny R. Mesenteric Castleman's disease: ultrasound, computed tomography and angiographic appearance. Australas Radiol. 2000;44(1):109-111. doi: 10.1046/j.1440-1673.2000.00753.x.

54. Yamashita Y, Hirai T, Matsukawa T, Ogata I, Takahashi M. Radiological presentations of Castleman's disease. Comput Med Imaging
Graph. 1993;17(2):107-117. doi: 10.1016/08956111(93)90052-o.

55. Tan TY, Pang KP, Goh HK, Teo EL, Abhilash B, Walford N. Castleman's disease of the neck a description of four cases on contrast-enhanced CT. Br J Radiol. 2004;77(915):253-256. doi: 10.1259/bjr/52051980.

56. Johnson WK, Ros PR, Powers C, Stoupis C, Segel $\mathrm{KH}$. Castleman disease mimicking an aggressive retroperitoneal neoplasm. Abdom Imaging. 1994;19(4):342-344. doi: 10.1007/ BF00198194.

\title{
Problems in the differential diagnosis of abdominal non-organ tumors
}

\author{
E.S. Kolobanova' • B.M. Medvedeva'
}

Rationale: The differential diagnosis between lesions requiring surgical intervention and those necessitating medical treatment poses a significant problem for current imaging methods of fat-containing masses in the small intestine mesenterium.

Aim: To identify differential diagnostic criteria for various fat-containing neoplasms of abdominal cavity at the preoperative stage.

Materials and methods: This retrospective study included data from 29 patients (16 women and 13 men, aged 32 to 81 years, mean age 56 years) with fat-containing masses in the small intestine mesenterium who had undergone preoperative examination at N.N. Blokhin National Medical Research Center of Oncology from 2016 to 2020. Abdominal computed tomography (CT) with intravenous contrast enhancement and assessment of the size, contours, structure and involvement of main vessels was performed in all patients. To interpret single soft tissue nodes within the mass, we used our own list of main CT symptoms (number, size, shape, contours and structure of nodes, degree of their contrast enhancement, and maximal amplification).

Results: Fat-containing abdominal masses were morphologically verified as follows: mesenteric panniculitis 13 (45\%), well differentiated liposarcoma $10 \quad(35 \%)$, lymphoproliferative disorders 4 (14\%), lipoma and Castleman disease 1 case each (3\%). Twelve (12) of 29 patients (41\%) had surgery for various types of tumors, while $17(59 \%)$ patients received appropriate medical treatment after a puncture biopsy-based morphological diagnosis. The CT sensitivity for the detection of fat-containing abdominal neoplasms in our series was $93,1 \%$. The paper describes the radiological signs of the above mentioned mass types with an emphasis on the complexity of their diagnosis.

Conclusion: In most cases, CT data can accurately differentiate abdominal liposarcomas from other benign and malignant masses in the small intestine mesenterium.

Key words: liposarcoma, mesenteric panniculitis, lymphoma, Castleman disease

For citation: Kolobanova ES, Medvedeva BM Problems in the differential diagnosis of abdominal non-organ tumors. Almanac of Clinical Medicine. 2021:49(1):29-40. doi: 10.18786/2072-0505-2021-49008.

Received 7 December 2020; revised 26 December 2020; accepted 1 March 2021; published online 15 March 2021

\section{Conflict of interests}

The authors declare no obvious and potential conflicts of interests related to the publication.

Authors' contributions

Both authors have equally contributed to the study conduct and preparation of the manuscript, have read and approved the final version of the manuscript before submission, consented to be accountable for all aspects of the work in ensuring that questions related to the accuracy or integrity of any part of the work are appropriately investigated and resolved.
Evgenia S. Kolobanova - MD, Radiologist, Department of Radiology, Scientific and Research Institute of Clinical and Experimental Radiology'; ORCID: https://orcid.org/0000-0002-1563-0983 $\triangle 23$ Kashirskoe shosse, Moscow, 115478, Russian Federation. Tel.: +7 (985) 1523039. E-mail: evgesha.16.kolobanova@gmail.com

Bela M. Medvedeva - MD, PhD, Leading Research Fellow, Department of Radiology, Scientific and Research Institute of Clinical and Experimental Radiology'; ORCID: https://orcid.org/0000-00031779-003X 